\title{
Research on Braking Electric Vehicle of Producing Renewable Energy
}

\author{
Zhi-qiang Xu \\ Guangdong University of Science \& Technology, Dongguan, Guangdong, 523083,china
}

Keywords: braking, electric vehicles, renewable energy.

\begin{abstract}
Every time a car's brake is applied a lot of energy is wasted. Physics tells teaches that energy cannot be destroyed. So when the car slows down, the kinetic energy that was propelling it forward has to go somewhere. Most of it simply dissipates as heat and becomes useless. That energy, which could have been used to do work, is essentially wasted.
\end{abstract}

\section{Introduction}

OrgaIs there anything that, the driver, can do to stop wasting this energy? Not really. In most cars it's the inevitable byproduct of braking and there's no way one can drive a car without occasionally hitting the brakes. But automotive engineers have given this problem a lot of thought and have come up with a kind of braking system that can recapture much of the car's kinetic energy and convert it into electricity, so that it can be used to recharge the car's batteries. This system is called regenerative braking.

At present, these kinds of brakes are primarily found in hybrid vehicles like the Toyota Prius, and in fully electric cars, like the Tesla Roadster. In vehicles like these, keeping the battery charged is of considerable importance. However, the technology was first used in trolley cars and has subsequently found its way into such unlikely places as electric bicycles and even Formula One race cars.

\section{Structures and working principle of a braking vehicles renewable energy}

In a traditional braking system, brake pads produce friction with the brake rotors to slow or stop the vehicle. Additional friction is produced between the slowed wheels and the surface of the road. This friction is what turns the car's kinetic energy into heat. With regenerative brakes, on the other hand, the system that drives the vehicle does the majority of the braking. When the driver steps on the brake pedal of an electric or hybrid vehicle, these types of brakes put the vehicle's electric motor into reverse mode, causing it to run backwards, thus slowing the car's wheels. While running backwards, the motor also acts as an electric generator, producing electricity that's then fed into the vehicle's batteries. These types of brakes work better at certain speeds than at others. In fact, they're most effective in stop-and-go driving situations. However, hybrids and fully electric cars also have friction brakes, as a kind of back-up system in situations where regenerative braking simply won't supply enough stopping power. In these instances, it's important for drivers to be aware of the fact that the brake pedal might respond differently to pressure. The pedal will sometimes depress farther towards the floor than it normally does and this sensation can cause momentary panic in drivers.

In the following pages, we'll take a more detailed look at how a regenerative braking system works, and we'll discuss reasons why regenerative braking is more efficient than a typical friction brake system.

In electric and hybrid cars, the regenerative brakes charge the main battery pack, effectively extending the vehicle's range between charges. Electric trains, which are powered by overhead or trackside power lines, work in a slightly different way. Instead of sending braking energy into batteries, they return it to the power line. A typical modern electric train can save around 15-20 percent of its energy using regenerative brakes in this way. Some vehicles use banks of super capacitors for storing energy instead of batteries.

Regenerative braking is used in vehicles that make use of electric motors, primarily fully electric vehicles and hybrid electric vehicles. One of the more interesting properties of an electric motor is 
that, when it's run in one direction, it converts electrical energy into mechanical energy that can be used to perform work (such as turning the wheels of a car), but when the motor is run in the opposite direction, a properly designed motor becomes an electric generator, converting mechanical energy into electrical energy. This electrical energy can then be fed into a charging system for the car's batteries.

In a regenerative braking system, the trick to getting the motor to run backwards is to use the vehicle's momentum as the mechanical energy that puts the motor into reverse. Momentum is the property that keeps the vehicle moving forward once it's been brought up to speed. Once the motor has been reversed, the electricity generated by the motor is fed back into the batteries, where it can be used to accelerate the car again after it stops. Sophisticated electronic circuitry is necessary to decide when the motor should reverse, while specialized electric circuits route the electricity generated by the motor into the vehicle's batteries. In some cases, the energy produced by these types of brakes is stored in a series of capacitors for later use. In addition, since vehicles using these kinds of brakes also have a standard friction braking system, the vehicle's electronics must decide which braking system is appropriate at which time. Because so much is controlled electronically in a regenerative braking system, it's even possible for the driver to select certain presets that determine how the vehicle reacts in different situations. For instance, in some vehicles a driver can select whether regenerative braking should begin immediately whenever the driver's foot comes off the accelerator pedal and whether the braking system will take the car all the way to $0 \mathrm{mph}$ ( 0 kilometers per hour) or will let the car coast slightly.

Brake controllers are electronic devices that can control brakes remotely, deciding when braking begins ends, and how quickly the brakes need to be applied. In towing situations, for instance, brake controllers can provide a means of coordinating the brakes on a trailer with the brakes on the vehicle doing the towing.

Regenerative braking is implemented in conjunction with anti-lock braking systems (ABS), so the regenerative braking controller is similar to an ABS controller, which monitors the rotational speed of the wheels and the difference in that speed from

One wheel to another. In vehicles that use these kinds of brakes, the brake controller not only monitors the speed of the wheels, but it can calculate how much torque -- rotational force -- is available to generate electricity to be fed back into the batteries. During the braking operation, the brake controller directs the electricity produced by the motor into the batteries or capacitors. It makes sure that an optimal amount of power is received by the batteries, but also ensures that the inflow of electricity isn't more than the batteries can handle.

The most important function of the brake controller, however, may be deciding whether the motor is currently capable of handling the force necessary for stopping the car. If it isn't, the brake controller turns the job over to the friction brakes, averting possible catastrophe. In vehicles that use these types of brakes, as much as any other piece of electronics on board a hybrid or electric car, the brake controller makes the entire regenerative braking process possible.

How is a hybrid vehicle different from a fully electric vehicle? Well, hybrid electric vehicles use both an electric motor and an internal combustion engine to provide a best-of-both-worlds driving experience. They combine the driving range of an internal combustion engine with the fuel efficiency and emissions-free characteristics of an electric motor. If a hybrid is to have maximum fuel efficiency and produce as few carbon emissions as possible, it's important that the battery remain charged as long as possible. If a hybrid vehicle battery were to lose its charge, the internal combustion engine would be entirely responsible for powering the vehicle. At that point, the vehicle is no longer acting as a hybrid but rather just another car burning fossil fuels.

Automotive engineers have come up with a number of tricks to wring the maximum efficiency out of hybrids, like aerodynamic streamlining of the bodies and use of lightweight materials, but arguably, one the most important is regenerative braking. In a hybrid setup, however, these types of brakes can provide power only to the electric motor part of the drivetrain via the vehicle's battery. The internal combustion Engine gains no advantage from these kinds of brakes. 


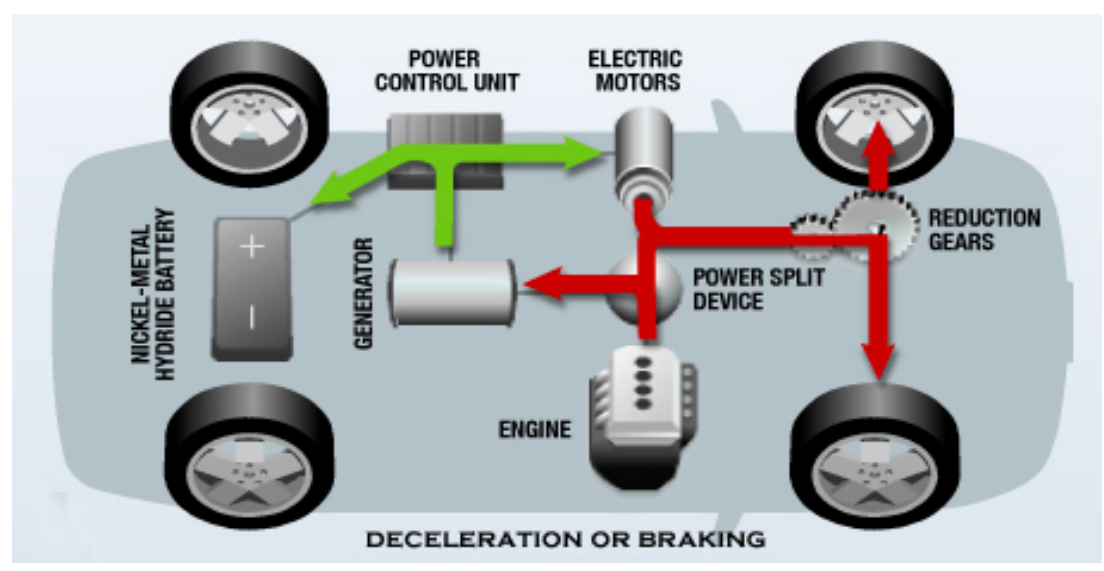

Fig. 1: Model electric generating vehicle

In part, these efficiencies are necessary due to the extreme difficulty in finding a place to recharge a hybrid. This makes longer trips difficult without relying on the hybrid's internal combustion engine, which actually cancels out some of the advantage of owning a hybrid.

Up next, we'll learn about a new take on this idea of regenerative braking.

An alternative regenerative braking system is being developed by the Ford Motor Company and the Eaton Corporation. It's called Hydraulic Power Assist or HPA. With HPA, when the driver steps on the brake, the vehicle's kinetic energy is used to power a reversible pump, which sends hydraulic fluid from a low pressure accumulator (a kind of storage tank) inside the vehicle into a high pressure accumulator. The pressure is created by nitrogen gas in the accumulator, which is compressed as the fluid is pumped into the space the gas formerly occupied. This slows the vehicle and helps bring it to a stop. The fluid remains under pressure in the accumulator until the driver pushes the accelerator again, at which point the pump is reversed and the pressurized fluid is used to accelerate the vehicle, effectively translating the kinetic energy that the car had before breaking into the mechanical energy that helps get the vehicle back up to speed. It's predicted that a system like this could store 80 percent of the momentum lost by a vehicle during deceleration and use it to get the vehicle moving again [source: HybridCars.com]. This percentage represents an even more impressive gain than what is produced by current regenerative braking systems. Like electronic regenerative braking, these kinds of brakes -- HPA systems -- are best used for city driving, where stop-and-go traffic is common.

\section{Quarry Equipment}

So far, HPA systems have been used primarily as proofs of concept and in demonstration projects only. They aren't quite ready for production models just yet. At present, these hydraulic brakes are noisy and prone to leaks; however, once all of the details are ironed out, such systems will probably be most useful in large trucks weighing 10,000 pounds (4,536 kilograms) or more, where these types of brakes may prove to be a more optimal system than electronically controlled regenerative brakes.

\section{Design of the technology}

Eventually, this technology may trickle down to smaller vehicles. One company, Hybrid-Drive Systems, LLC, of Michigan, has retrofitted a 1968 Volkswagen Beetle with a hydraulic regenerative braking system. However, the accumulators take up a considerable amount of space, and future production plans are focused more on using the technology in larger vehicles, like vans. Meanwhile, the U.S. Environmental Protection Agency (EPA) has partnered with Eaton Corporation to install hydraulic regenerative braking systems on UPS delivery trucks.

\section{Design of the energy efficiency of a braking vehicle}

The energy efficiency of a conventional car is only about 20 percent, with the remaining 80 percent of its energy being converted to heat through friction. The miraculous thing about 
regenerative braking is that it may be able to capture as much as half of that wasted energy and put it back to work. This could reduce fuel consumption by 10 to 25 percent. Hydraulic regenerative braking systems could provide even more impressive gains, potentially reducing fuel use by 25 to 45 percent [source: HybridCars.com]. In a century that may see the end of the vast fossil fuel reserves that have provided us with energy for automotive and other technologies for many years, and in which fears about carbon emissions are coming to a peak, this added efficiency is becoming increasingly important.

\section{Design of renewable energy}

The beginning of the 21st century could very well mark the final period in which internal combustion engines are commonly used in cars. Already automakers are moving toward alternative energy carriers such as electric batteries, hydrogen fuel and even compressed air.

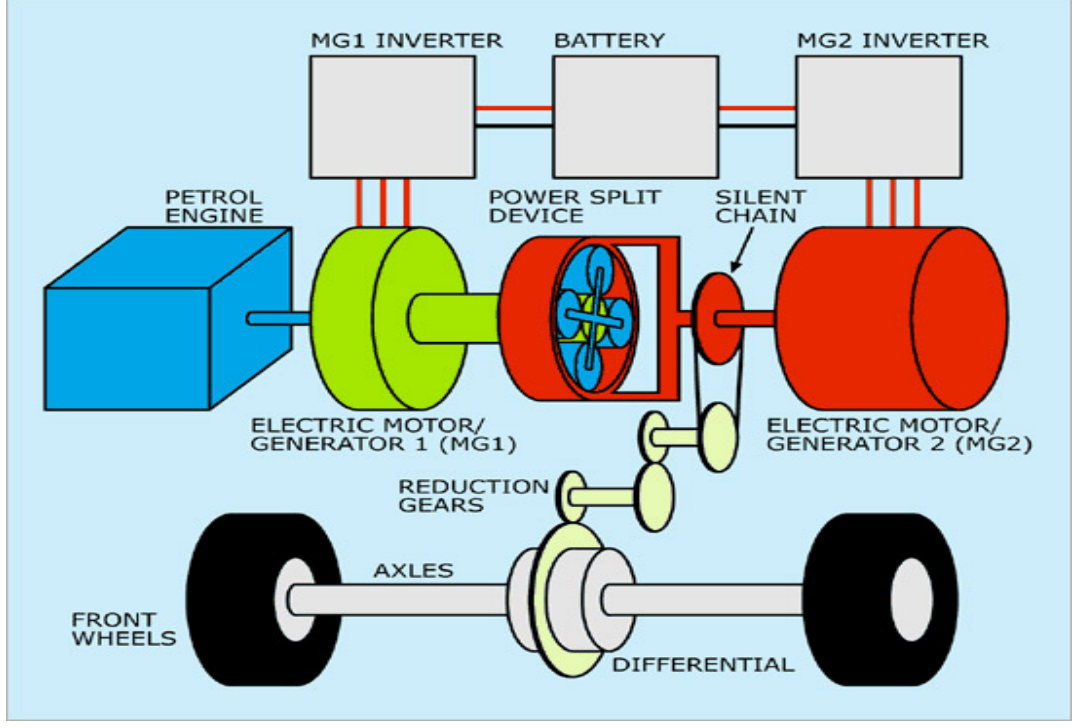

fig.2 Process of generation of energy by vehicle.

Regenerative braking is a small, yet very important, step toward our eventual independence from fossil fuels. These kinds of brakes allow batteries to be used for longer periods of time without the need to be plugged into an external charger. These types of brakes also extend the driving range of fully electric vehicles. In fact, this technology has already helped bring us cars like the Tesla Roadster, which runs entirely on battery power. Sure, these cars may use fossil fuels at the recharging stage -that is, if the source of the electricity comes from a fossil fuel such as coal but when they're out there on the road, they can operate with no use of fossil fuels at all, and that's a big step forward.

\section{Summary}

The added efficiency of regenerative braking also means less pain at the pump, since hybrids with electric motors and regenerative brakes can travel considerably farther on a gallon of gas, some achieving more than 50 miles per gallon at this point. And that's something that most drivers can really appreciate.

\section{References}

[1]Jeff Cobb . "December 2014 Dashboard". HybridCars.com and Baum \& Associates(2015-01-06). [2]Matthe, Roland; Eberle, Ulrich. "The Voltec System - Energy Storage and Electric Propulsion" (2014-01-01).

[3]Stensvold, Tore. "Lønnsomt å bytte ut 70 prosent av fergene med batteri- eller hybridferger" Teknisk Ukeblad. 